\title{
Solution Properties of Synthetic Polypeptides. XIV. Synthesis and Characterization of Broken-rod Polypeptides
}

\author{
Koichi Nakagawa, ${ }^{*}$ Noboru Nishioka, Akio Teramoto, \\ and Hiroshi FujITA \\ Department of Polymer Science, Osaka University, Toyonaka, Osaka 560, Japan.
}

(Received June 21, 1972)

\begin{abstract}
Attempts were made to obtain model compounds for once-broken rod molecules by two different methods. In one of them, $N$-carboxy anhydride of $\gamma$-benzyl glutamate (BG-NCA) was polymerized in dimethylformamide (DMF) containing trimethylenediamine (TMDA) as an initiator. In the other, BLG-NCA was polymerized in DMF with the preformed polymer of BDG-NCA as an initiator. It is expected that the molecule obtained by the first method yields a polymer chain which contains the TMDA residue somewhere in its central portion, whereas the second method gives a block copolymer of the DL type. The initiator residue in the former and the boundary between the $\mathrm{L}$ and $\mathrm{D}$ chains in the latter are intolerant of helix formation even in helicogenic solvents, and they should act as flexible joints of two helical rods. As an additional attempt, BLG-NCA was polymerized in DMF containing the polymer preformed by TMDA-initiated polymerization of BDG-NCA, in the hope of synthesizing a block copolymer of the LDL type. This polymer is expected to assume the form of a thrice-broken rod when dissolved in a helicogenic solvent. The postulated structures of the polypeptides thus obtained were confirmed by measurements of polymerization rate and optical rotation. It was difficult to prepare high-molecularweight samples of the desired broken-rod structure by the procedures employed.
\end{abstract}

KEY WORDS: Poly( $\gamma$-benzyl glutamate) / Once-broken Rod / LD and LDL- Block Copolymers / Polymerization Kinetics / Helix-coil Transition / Optical Rotatory Dispersion /

Model compounds for once-broken rod molecules may be synthesized either by polymerizing an $N$-carboxy- $\alpha$-amino acid anhydride (NCA) with a diamine as an initiator or by blockcopolymerizing the $\mathrm{L}$ and $\mathrm{D}$ forms of an NCA. In the polymer obtained by the first method, it is expected that the diamine residue is incorporated somewhere in the central part of the chain and will act as a flexible joint connecting two polypeptide chains. On the other hand, in the DL block copolypeptide, a few monomer units located in the boundary between the $\mathrm{L}$ and $\mathrm{D}$ chains will play a similar role, because for steric reasons they must assume a disordered structure even in helicogenic solvents.

In Part IV $^{1}$ of this series, an attempt was made to polymerize $\gamma$-benzyl L-glutamate NCA

\footnotetext{
* Press address: Electrical Communication Laboratory, Nippon Telegraph and Telephone Public Corporation, Ibaragi, Japan.
}

(BLG-NCA) in dioxane containing trimethylenediamine (TMDA) as an initiator. We have reattempted TMDA initiated polymerization of $\gamma$-benzyl glutamate NCA (BG-NCA), this time using dimethylformamide (DMF) as a solvent on the basis of the finding by Lundberg and Doty $^{2}$ that polymerization of BLG-NCA initiated by a primary amine, when carried out in this solvent, obeyed a pseudo-first-order reaction scheme and produced samples having very narrow distribution of molecular weight.

Gratzer and Doty ${ }^{3}$ developed a procedure for preparing block copolypeptides, in which an NCA is polymerized with a preformed polymer of another NCA as an initiator, the idea first suggested by Hanby, et al. ${ }^{4}$ The mechanism and kinetics of polymerization of BDG-NCA with preformed BLG polymers in DMF were investigated by Lundberg and Doty. ${ }^{2}$ In the present study, we have undertaken to synthesize 
DL and LDL block copolymers of BG by using the Gratzer-Doty method.

Kinetics of the formation of these polypeptides and optical rotatory properties of the products were examined in order to ascertain whether the postulated structures were obtained. A preliminary account of the present work had been presented elsewhere. ${ }^{5}$

\section{EXPERIMENTAL}

\section{Solvents}

Thoroughly purified organic compounds were used for both preparative work and physical measurements.

\section{Polypeptides}

The monomer, $N$-carboxy anhydride of $\gamma$ benzyl glutamate (written BG-NCA), was prepared according to the method reported by Blout and Karlson. ${ }^{6}$ Polymerization of it was carried out in DMF of $25^{\circ} \mathrm{C}$ or of a temperature close to it, by the procedures described below.

$P B G-A$. This was prepared by polymerization of BLG-NCA with $n$-butylamine as an initiator. The resulting polymer attaches the initiator residue to its end, ${ }^{2,7-10}$ and ought to assume the form of a straight rod in helicogenic solvents such as DMF.

$P B G-B$. This was synthesized by polymerizing BLG (or BDG)-NCA with TMDA as an initiator. For the reason mentioned above the resulting polymer should behave like a oncebroken rod when dissolved in a helicogenic solvent.

$P B G-C$. First, BDG-NCA was polymerized with $n$-butylamine as an initiator. At the stage where the added NCA was almost consumed, BLG-NCA was introduced into the solution by the amount equal to that of the initially added BDG-NCA, and the mixture was allowed to stand. According to well-established evidence, ${ }^{2,7-10}$ the amino residue at the growing end of each preformed PBDG chain maintains activity so that it can initiate polymerization of the L-NCA added. Thus, during the period of standing, a PBLG chain should grow from one end of each preformed $D$ chain to form a DL block copolymer of BG. Recently, a similar
Table I. Preparative data of $\operatorname{poly}(\gamma$-benzyl glutamate) samples

\begin{tabular}{rcrrrr}
\hline $\begin{array}{c}\text { Sample } \\
\text { code }\end{array}$ & $\begin{array}{c}\text { Type } \\
\text { NCA } \\
\text { NCA }\end{array}$ & $\begin{array}{c}\text { Concn } \\
\text { of } \\
\text { NCA, } \\
\text { wt } \%\end{array}$ & $\begin{array}{c}\text { [A }]_{0} /[\mathrm{I}]_{0}, \\
\text { (mole }\end{array}$ & $\bar{M}_{v} \times 10^{-4}$ & $\begin{array}{c}\text { Yield, } \\
\%\end{array}$ \\
\hline PCG A-1 & L & 5 & 10 & 0.347 & 89 \\
A-2 & L & 5 & 20 & 0.476 & 96 \\
A-3 & L & 5 & 50 & 1.06 & 85 \\
A-4 & L & 5 & 100 & 1.42 & 76 \\
A-5 & L & 8 & 300 & 2.30 & 93 \\
A-6 & L & 10 & 1000 & 3.70 & 85 \\
B-1 & D & 5 & 50 & 1.10 & 88 \\
B-2 & D & 5 & 100 & 1.57 & 65 \\
B-3 & D & 10 & 300 & 3.70 & 88 \\
B-4 & D & 10 & 1000 & 6.58 & 90 \\
C-1 & D+L & 5 & 20 & 0.80 & 83 \\
C-2 & D+L & 5 & 50 & 1.29 & 91 \\
C-3 & D+L & 5 & 100 & 1.90 & 80 \\
C-4 & D+L & 5 & 200 & 2.86 & 87 \\
D-1 & D+L & 5 & 40 & 0.96 & 80 \\
D-2 & D+L & 5 & 200 & 2.26 & 88 \\
\hline
\end{tabular}

a Actual yields would be larger than those given here, since there was loss of polypeptide when extracting from the polymerization mixtures.

idea was used by Shoji and Kawai ${ }^{11}$ to prepare a DL block copolymer of alanine.

$P B G-D$. BLG-NCA may be polymerized by using a PBDG-B molecule, instead of a PBDG-A molecule, as the initiator. The resulting polymer, which is referred to as PBG-D in the subsequent presentation, ought to be a block copolymer of the LDL type. Since the TMDA residue incorporated in the $D$ block and the two boundary regions of the $\mathrm{D}$ and $\mathrm{L}$ blocks are intolerant of helix formation, this block copolypeptide is expected to behave like a thrice-broken rod in helicogenic solvents.

Table I summarizes the preparative data for the four kinds of polypeptide thus obtained.

\section{Polymerization Rate}

Polymerization kinetics of PBG-A, PBG-B, PBG-C, and PBG-D were examined according to the method of Lundberg and Doty. ${ }^{2}$ A weighed amount of NCA was dissolved in DMF, which was mixed with a DMF solution of the initiator in a reaction tube of about $2 \mathrm{~m} l$ capacity, and the $\mathrm{CO}_{2}$ gas evolved as the result of polymerization was swept out by a 
stream of dry nitrogen and absorbed into an 0.04- $N$ solution of $\mathrm{Ba}(\mathrm{OH})_{2}$ placed in a conductance cell. The resulting change in electric conductance of the $\mathrm{Ba}(\mathrm{OH})_{2}$ solution was followed by use of a Kohlrausch bridge. The experiments were carried out at molar ratios of NCA to initiator varying from 20 to 60 , with the molar concentration of added NCA, $[\mathrm{A}]_{0}$, being fixed at about $0.17 \mathrm{~mol} / l$.

\section{Optical Rotation}

For PBG-A and PBG-B in two mixtures of dichloroacetic acid (DCA) and ethylene dichloride (EDC) of volume ratios 65 (DCA) : 35 (EDC) and 76 (DCA) : 24 (EDC), both at $25^{\circ} \mathrm{C}$, the specific rotation for light of the wavelength $578 \mathrm{~nm}$, $[\alpha]_{578}$, was measured at temperatures from 20 to $60^{\circ} \mathrm{C}$. A Perkin-Elmer Model 141 polarimeter and a JASCO Model DIP_SL automatic polarimeter were used for this purpose.

Optical rotatory dispersions of several samples of PBG-A and PBG-B in DMF of $20^{\circ} \mathrm{C}$ were measured in the range of wavelength from 275 to $600 \mathrm{~nm}$ by use of a Yanagimoto Model ORD-181 recording spectropolarimeter. For comparison, the data were also taken with a PBLG sample (code A-VI, $\bar{M}_{w}=2.42 \times 10^{5}$ ) prepared previously in our laboratory. ${ }^{12}$ The densities and refractive indices needed for the analysis of ORD data were evaluated as described elsewhere. ${ }^{1,13}$ In all cases studied, the polymer concentrations were adjusted to $0.7-$ $1.2 \mathrm{~g} / \mathrm{d} l$.

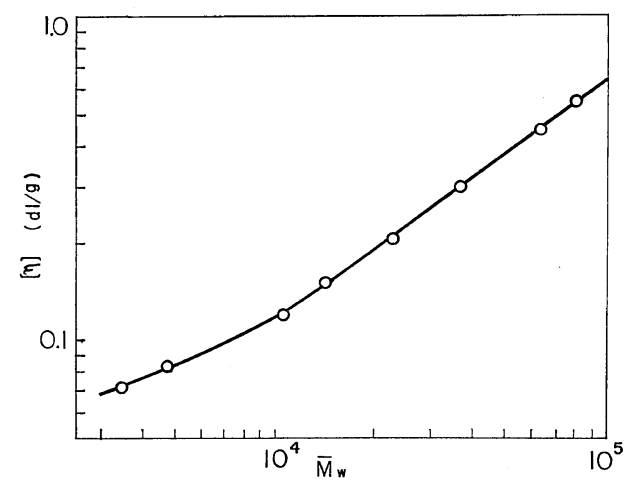

Figure 1. Intrinsic viscosity-molecular weight relationship for low molecular weight poly $(\gamma$-benzyl L-glutamate) in dichloroacetic acid at $25^{\circ} \mathrm{C}$. The weight-average molecular weights $\bar{M}_{w}$ were determined by sedimentation methods. ${ }^{14}$

\section{Molecular Weight}

Weight-average molecular weights, $\bar{M}_{w}$, of several PBG-A and PBG-B samples were determined by the Archibald method or by the sedimentation equilibrium method, with DMF of $25^{\circ} \mathrm{C}$ being used as a solvent. For all samples listed in Table I their intrinsic viscosities in DCA of $25^{\circ} \mathrm{C}$ were measured, and the results were converted to viscosity-average molecular weight, $\bar{M}_{v}$, with the acid of the relationship $^{14}$ shown in Figure 1.

\section{RESULTS AND DISCUSSION}

\section{Reaction Kinetics}

If a primary amine-initiated polymerization of NCA proceeds ideally, i.e., the initiation step is much faster than the propagation step and, moreover, the latter proceeds without termination, it follows that the molar concentration of NCA at time $t$ from the start of reaction is given by eq 1 characteristic of firstorder reaction: ${ }^{2,4,6-9,15}$

$$
\log \left([\mathrm{A}]_{0} /[\mathrm{A}]_{t}\right)=0.4343[\mathrm{I}]_{0} k_{2} t
$$

where $[A]_{0}$ and $[I]_{0}$ are the molar concentrations of NCA and initiator, at $t=0$, respectively, and $k_{2}$ is the rate constant for the propagation step. This equation can be transformed to the statement that plots of $\log \left([\mathrm{A}]_{0} /[\mathrm{A}]_{t}\right)$ vs. $t$ follow a straight line passing through the origin and

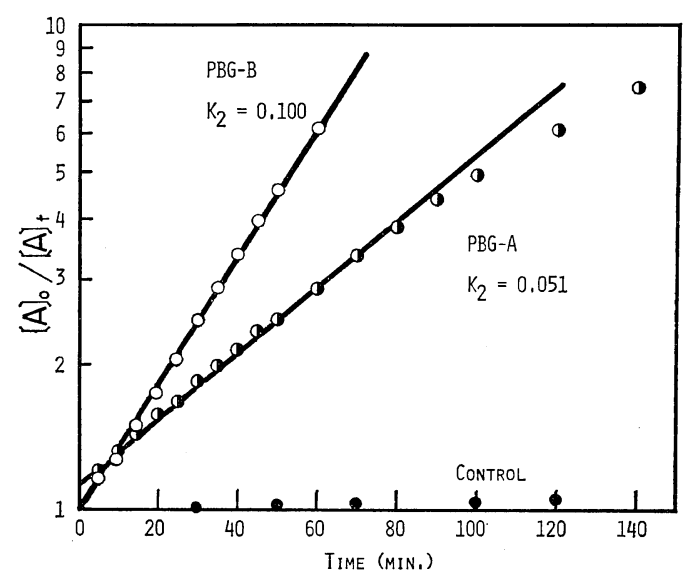

Figure 2. Kinetic curves for polymerization of $\mathrm{BG}-\mathrm{NCA}$ 's in dimethylformamide at $25^{\circ} \mathrm{C}$; [A $]_{0}$ $=0.22 \mathrm{~mol} / l$ and $[\mathrm{A}]_{0} /[\mathrm{I}]_{0}=40$ for PBG-A; $[\mathrm{A}]_{0}=$ $0.18 \mathrm{~mol} / l$ and $[\mathrm{A}]_{0} /[\mathrm{I}]_{0}=40$ for PBG-B. 
that, with the value of $[\mathrm{I}]_{0}$ known separately, one can evaluate $k_{2}$ from the slope of the line. In the subsequent presentation, values of $k_{2}$ are given in units of liter $\mathrm{mol}^{-1} \mathrm{sec}^{-1}$.

$P B G-A$ and $P B G-B$. Figure 2 shows tests of eq 1 with typical kinetic data on PBG-A and PBG-B. It is seen that, for either polymer, the data points fall on a straight line over a range of time, as predicted by eq 1 . The $k_{2}$ value derived from the line for PBG-A is 0.051 , whicn agrees closely with the value 0.056 reported by Lundberg and Doty ${ }^{2}$ for $n$-hexylamineinitiated polymerization of BLG-NCA in DMF at $25^{\circ} \mathrm{C}$. The filled circles in the figure are for a control in which no initiator was added, indicating that practically no polymerization took place in the absence of the initiator. The line for PBG-B gives 0.100 for $k_{2}$, which is nearly twice as large as the $k_{2}$ value obtained for PBG-A. This result may be taken to mean that polymer chains grow from the two amino residues of TMDA, each at a rate characteristic of a monoamine-initiated polymerization.

Kinetic data obtained under other conditions all gave similar results; i.e., the yield of polypeptide determined by the conductometric method exceeded $90 \%$, and the rate constants $k_{2}$ were, on the average, $0.054( \pm 0.004)$ for PBG-A and $0.01( \pm 0.10)$ for PBG-B.

$P B G-C$ and $P B G-D$. Typical kinetic data for the formation of PBG-C are illustrated in
Figure 3. It is seen that $\mathrm{CO}_{2}$ gas started evolving immediately after the L-NCA was added to the solution containing the preformed PBDG molecules. The two portions of the curve, one for the polymerization of D-NCA and the other for the copolymerization of L-NCA with the preformed $\mathrm{PBDG}$, are separately replotted in Figure 4, according to eq 1 . For both kinetic processes the experimental data appear to obey eq 1. The copolymerization rates $k_{2}$ for this and other cases, in which a different amount of L-NCA was added to the solution or in which copolymerization of L-NCA with the preformed PBG-B chains was pursued, are plotted against $D / L$, the amount of $D-N C A$ relative to that of L-NCA copolymerized, in Figure 5, where the value derived from the data of Lundberg and Doty $^{2}$ (filled circle) is indicated for comparison. One can observe that the values of $k_{2}$ may be extrapolated linearly to $D / L=0$ to obtain 0.055 \pm 0.005 for sufficiently large amount of L-NCA added. Interestingly, this limiting value is nearly equal to the $k_{2}$ value obtained above for the formation of PBG-A.

As has been pointed out by Lundberg and Doty, ${ }^{2}$ however, one has no reason to consider that the block copolymerization of L-NCA with the preformed $\mathrm{D}$ chain should follow a firstorder reaction represented by eq 1 . Instead, the slope of the plot for this process, such as shown in Figure 4, should be regarded as a

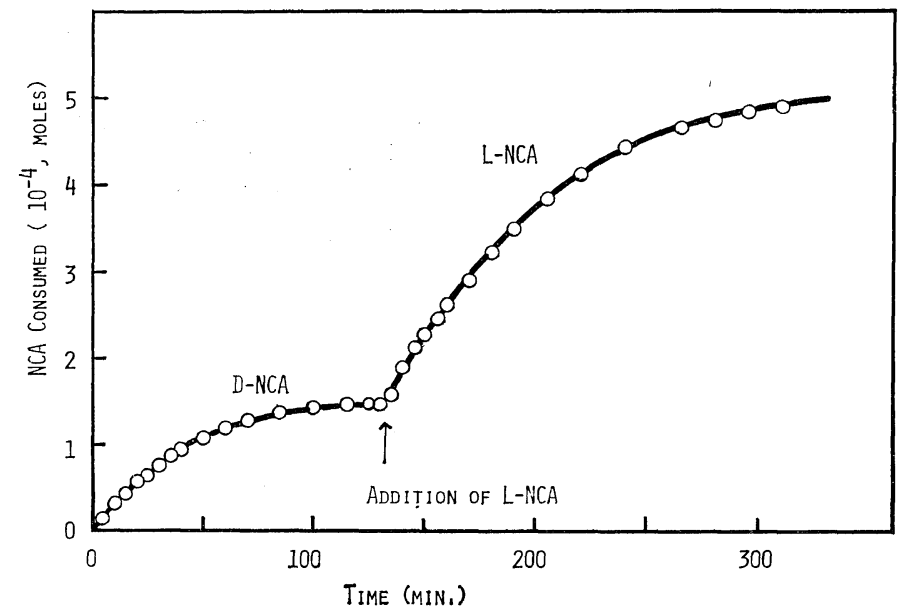

Figure 3. Kinetic curve for block copolymerization of $B L G-N C A$ and BDGNCA with $n$-butylamine initiation in DMF. 


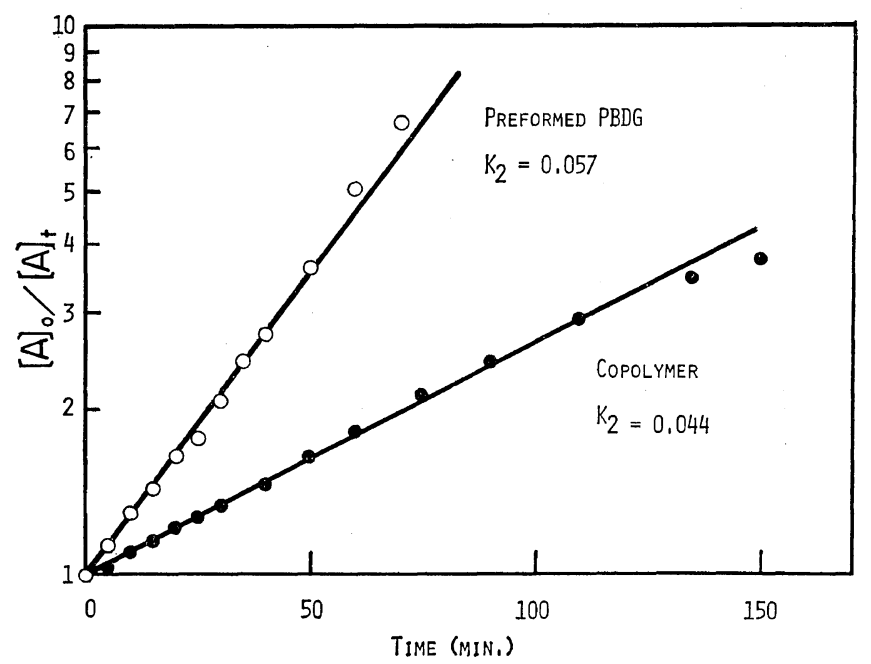

Figure 4. $\log$ of ratio of original anhydride concentration to that at time $t v s$. time with the data shown in Figure $3 ;[\mathrm{A}]_{0} /[\mathrm{I}]_{0}=20$ for the preformed $\mathrm{D}$ polymer and $[\mathrm{A}]_{0} /[\mathrm{I}]_{0}=60$ for the copolymerization of $\mathrm{L}-\mathrm{NCA}$.

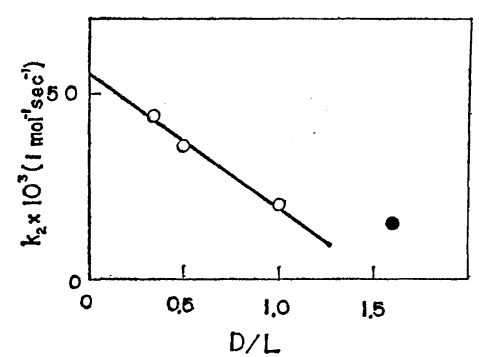

Figure 5. Average rate of copolymerization in DMF vs. amount of $\mathrm{D}-\mathrm{NCA}$ relative to that of L-NCA (see text). The filled circle denotes the value calculated from the data of Lundberg and Doty. ${ }^{2}$

certain average rate of the complex reactions involved. According to these authors, there will be a slow addition of L-NCA to the active ends of the preformed $D$ chains before the $L$ chains begin to form helices of their preferred screw sense and grow at a faster rate. Thus, the average rate of the copolymerization process is expected to be slower in the presence of smaller amounts of added L-NCA and become faster as more L-NCA is added. For sufficiently large amounts of L-NCA present the faster addition of the monomer to the growing $L$ chains will eventually dominate the whole course of copolymerization of the same NCA. These predictions agree with the actual observations described above.

Summarizing, we may conclude that our kinetic data are compatible with the molecular structures postulated for PBG-A, PBG-B, PBG-C, and PBG-D on the basis of the ideas underlying their methods of synthesis.

\section{Effect of $[A]_{0} /[I]_{0}$ on Molecular Weight}

The effect of $[\mathrm{A}]_{0} /[\mathrm{I}]_{0}$ on the molecular weight of the polymerization product is illustrated in Figure 6, wherein the data of Lundberg and Doty $^{2}$ for $n$-hexylamine-initiated polymerization of BLG-NCA in DMF are indicated for comparison. It is proper to use $\bar{M}_{n}$, the numberaverage molecular weight, for rigorous discussion of this effect, but because of cumbersomeness of measuring $\bar{M}_{n}$ by osmometry, we had to be content with replacing $\bar{M}_{n}$ by the more easily measurable $\bar{M}_{v}$.

If the ideal reaction mechanism underlying eq 1 is obeyed, the product should have a very narrow distribution of molecular weight represented by Poisson's formula, and its number-average degree of polymerization, $\bar{N}_{n}$, is given by the value of $[\mathrm{A}]_{0} /[\mathrm{I}]_{0}$ used for its synthesis. ${ }^{2,4,-10,15}$ In the approximation that $\bar{M}_{n}$ may be replaced by $\bar{M}_{v}$, this latter pre- 
Synthesis and Characterization of Broken-rod Polypeptides

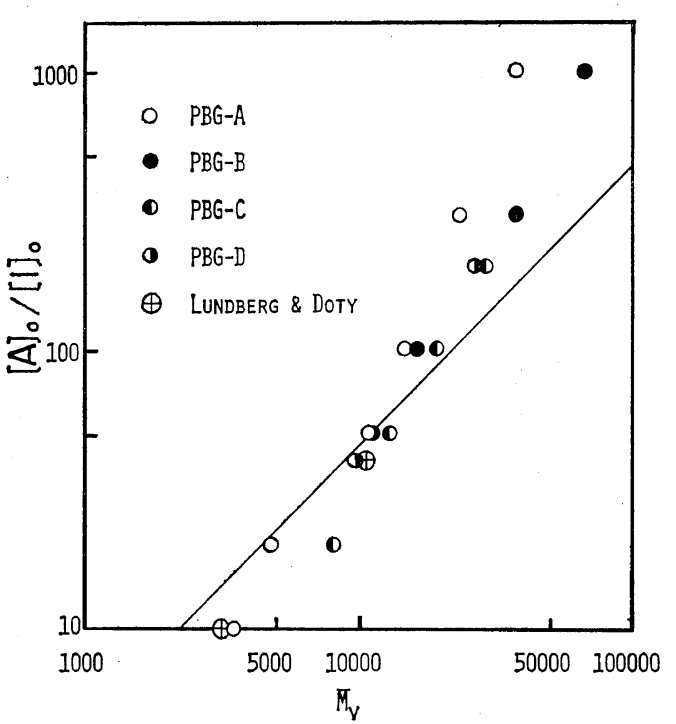

Figure 6. Effect of anhydride-initiator ratio on molecular weight for primary amine-initiated polymerization of BG-NCA in DMF. The data of Lundberg and Doty ${ }^{2}$ (with $n$-hexylamine initiation) are included for comparison.

diction is represented by the solid line shown in Figure 6. It is seen that the data come close to this ideal line for $[\mathrm{A}]_{0} /[\mathrm{I}]_{0}$ smaller than 100 but increasingly deviate above the line for the larger values of $[\mathrm{A}]_{0} /[\mathrm{I}]_{0}$, as was observed by Blout and Karlson ${ }^{6}$ in primary amine-initiated polymerization of NCA. Various causes may be considered for this type of deviation. They include contamination of the monomer with impurities, presence of "active" impurities in the solvent, and termination reactions. Here by "active" impurities we mean foreign chemical species which are capable of initiating polymerization of NCA. From a careful survey of published results Szwarc $^{9}$ concluded that the last one of the above causes is usually negligible, though it cannot be entirely ruled out. As far as our present data are concerned, the second one seems to have been a prime suspect, because it is quite probable that the DMF used as our solvent was still impure, though considerable efforts were made for its purification. This idea is consistent with the upward deviation from ideality shown in Figure 6, since the presence of active impurities always lowers the effective value of $[\mathrm{A}]_{0} /[\mathrm{I}]_{0}$ below that calcu- lated from the concentration of the externally added initiator.

Polymerization initiated by "active" impurities will produce only molecules of the PBG-A type. It is probable, therefore, that a sample of the broken-rod type prepared in an impure solvent will contain a certain fraction of PBG molecules of this type. For this reason we suspect that our broken-rod samples obtained at higher values of $[\mathrm{A}]_{0} /[\mathrm{I}]_{0}$ suffered such a contamination, although, at present, no idea on how to estimate its degree experimentally occurs to us.

In the case of synthesizing PBG-A, regardless of whether the solvent contains "active" impurities or not, the resulting sample should have a very narrow distribution of molecular weight if the polymerizations initiated by both added initiator and "active" impurities proceed ideally. In fact, the $\bar{M}_{w} / \bar{M}_{n}$ for sample A-6 is as close to unity as $1.16^{13}$ and that for sample A-4 is much closer to unity, ${ }^{16}$ despite the solvent used for its synthesis is supposed to have been considerably contaminated with "active impurities, if judged from the location of the data point for each sample in Figure 6.

\section{Optical Rotation}

Thermally-induced Helix-coil Transition. Data for $[\alpha]_{578}$ as a function of temperature are shown in Figures 7 and 8 . With the understanding that $[\alpha]$ at a specified wavelength is linearly related to the helical content of the dissolved (homo)polypeptide, these graphs can be taken to represent the processes in which PBG-A and PBG-B change their conformation from random coil to helix as the temperature is raised. One of the interesting features is that the transition curve is markedly flattened with decreasing molecular weight of the sample. This behavior can be interpreted in terms of the current theories of helix-coil transitions in polypeptide. $^{17}$

If the two chains separated by the TMDA residue in a PBG-B molecule are equal in length and if, as is probably the case, they can take part in helix formation without mutual interference, it follows that the molecule should behave polarimetrically as if it were a PBG-A molecule having a chain length of its half. 
K. Nakagawa, N. Nishioka, A. Teramoto, and H. Fujita

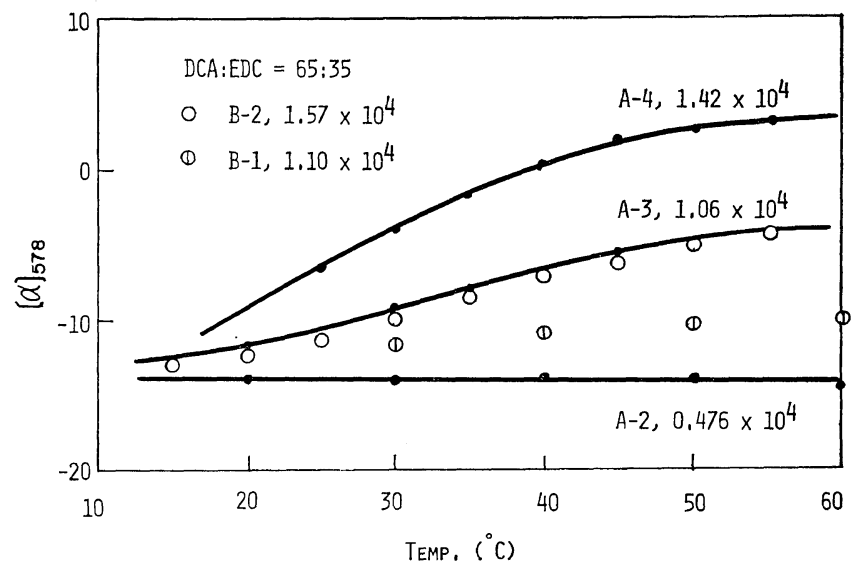

Figure 7. Specific rotation for light of wavelength $578 \mathrm{~nm},[\alpha]_{578}$, for samples of PBG-A and PBG-B in a mixture of DCA and EDC (volume ratio of $25^{\circ} \mathrm{C}=65$ : 35). Solid lines represent the data for PBG-A samples and circles are those of PBG-B, with their signs being reversed in the latter case.

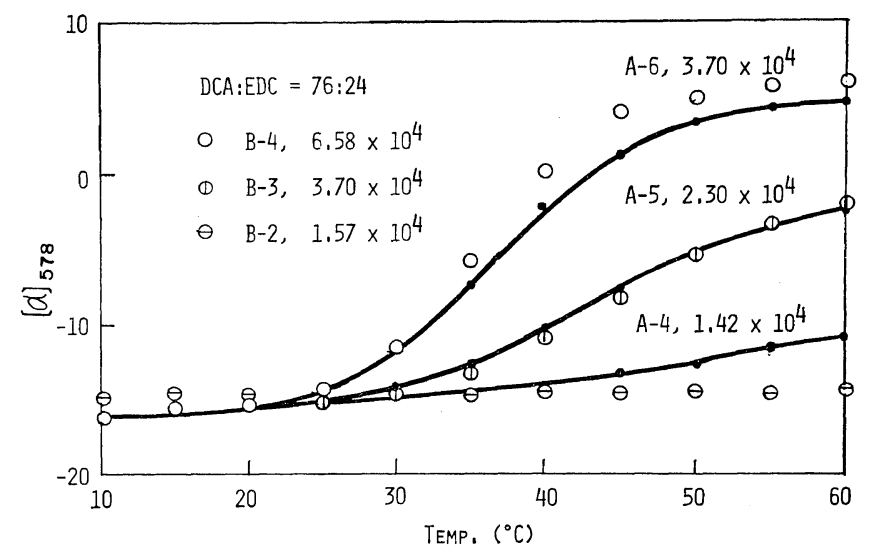

Figure 8. Specific rotation $[\alpha]_{578}$ for samples of PBG-A and PBG-B in a mixture of DCA (76) and EDC (24). The data are presented in the same way as in Figure 7.

Qualitative verification of this prediction is presented in Figures 7 and 8 , in which the transition curves of several PBG-B samples are seen nearly to coincide with those of PBG-A samples whose $\bar{M}_{v}$ 's are approximately half the $\bar{M}_{v}$ values of the corresponding PBG-B samples.

Optical Rotatory Dispersion in Helicogenic Solvent DMF. ORD data obtained for PBG-A and PBG-B in the helicogenic solvent DMF were analyzed in terms of Moffitt's equation, with the parameter $\lambda_{0}$ being taken to be 212 $\mathrm{nm}$. The results are summarized in Table II.
Table II. Moffitt parameters $a_{0}$ and $b_{0}$ for PBG-A and PBG-B in DMF of $20^{\circ} \mathrm{C}^{\mathrm{a}}$

\begin{tabular}{cccr}
\hline Sample code & $\bar{N}_{v}$ & $b_{0}$ & $a_{0}$ \\
\hline PBG A-1 & 15.8 & $-325 \pm 10$ & 105 \\
A-2 & 21.6 & $-456 \pm 10$ & 148 \\
A-3 & 48.4 & $-590 \pm 15$ & 155 \\
A-6 & 169 & $-670 \pm 10$ & 157 \\
A-VI & 1100 & $-675 \pm 10$ & 160 \\
B-1 & 50 & $494 \pm 10$ & -145 \\
B-2 & 71.7 & $583 \pm 10$ & -160 \\
B-3 & 169 & $650 \pm 10$ & -170 \\
\hline
\end{tabular}

a $\lambda_{0}$ taken to be $212 \mathrm{~nm}$ as usual. 
For either PBG-A or PBG-B the absolute value of the Moffitt parameter $b_{0}$ decreases markedly as the molecular weight of the sample is lowered. When compared at the same molecular weight, this value for PBG-B is consistently smaller than that for PBG-A.

One may consider that, in the helicogenic solvent DMF, all the chain units of a PBG-A molecule will participate in helix formation, except for a few located in the vicinity of the chain ends. Hence, if the number of such units is denoted by $N_{\mathrm{c}}$, the helical content $f_{N}$ of a PBG-A sample in DMF may be represented by

$$
f_{N}=\left(N-N_{\mathrm{c}}\right) / N=1-\left(N_{\mathrm{c}} / N\right)
$$

where $N$ is the degree of polymerization of the sample. In the case of PBG-B, not only those monomer units near the chain ends but also a few units adjacent to the TMDA residue incorporated in the molecule will not take part in helix formation. Therefore, the helical content of a PBG-B sample in DMF is also represented by eq 2 if $N_{\mathrm{c}}$ is taken to be the total number of such units intolerant of helix formation. It is legitimate to consider that the value of $N_{\mathrm{c}}$ is larger for PBG-B than for PBG-A.

With the usual assumption that $b_{0}$ is proportional to $f_{N}$ under fixed solvent conditions, eq 2 may be rewritten

$$
b_{0}=\left(b_{0}\right)_{*}-\left(b_{0}\right)_{*} N_{\mathrm{c}} / N
$$

where $(b)_{*}$ denotes the value of $b_{0}$ which would be obtained in a helicogenic solvent if the molecular weight of the sample were infinitely large. This equation predicts that plots of $b_{0}$ against $1 / N$ should follow a straight line if $N_{\mathrm{c}}$ is independent of $N$. Furthermore, from the definition of $\left(b_{0}\right)_{*}$ one can expect that an identical value should be obtained for this quantity from the data for both PBG-A and PBG-B. Tests of these predictions with the data given in Table II are displayed in Figure 9, wherein $\bar{N}_{v}$ is used for $N$. As expected, the straight lines for both polymers converge to the same ordinate intercept, yielding $-700 \pm 10$ for $\left(b_{0}\right)_{*}$. On the other hand, the slopes of the straight lines in this figure give $7 \pm 1$ for $N_{\mathrm{c}}$ of PBG-A and $14 \pm 1$ for that of PBG-B.

The value of $N_{\mathrm{c}}$ for PBG-A is consistent with the conclusion of previous authors ${ }^{2,7}$ from ORD and reaction kinetics that the minimum degree of polymerization for which the molecule of PBG can maintain $\alpha$-helix is in the range 7-14. As a further test of eq 3, we chose the data reported by Goodman, et al.,$^{18}$ for poly $(\gamma$-methyl L-glutamate) in DMF and obtained 6.2 for $N_{\mathrm{c}}$. Again, this value is compatible with their conclusion that helix formation occurs first at the pentapeptide and becomes distinctive at the nonapeptide.

The value obtained for $N_{\mathrm{c}}$ of PBG-B is about

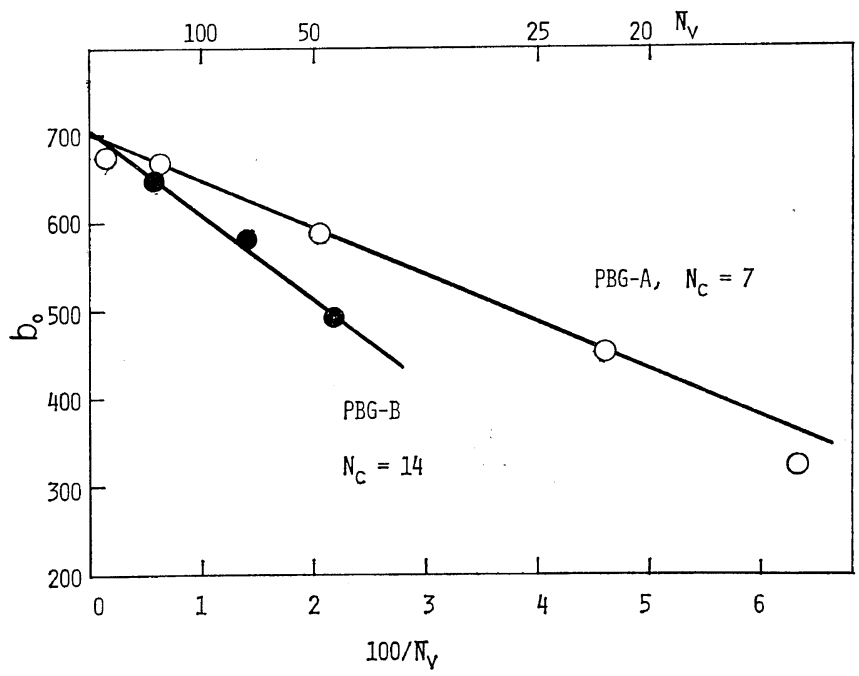

Figure 9. Dependence of Moffitt parameter $b_{0}$ on the degree of polymerization for $\mathrm{PBG}$ 's in DMF of $20^{\circ} \mathrm{C}$. For PBG-A, the values of $-b_{0}$ are plotted. 
twice as large as that of PBG-A, implying that, in the molecule of PBG-B, approximately equal numbers of monomer units adjacent to the chain ends at the TMDA residue are intolerant of helix formation under this solvent condition. Or it may be taken as evidence showing that PBG-B contains a TMDA residue somewhere in its middle portion, as can be expected from the bifunctionality of this diamine.

\section{Concluding Remarks}

Our results on polymerization kinetics, thermally-induced helix-coil transition, and optical rotatory dispersion have all lent support to the idea that a model compound for a once-broken rod molecule can be obtained by polymerizing BG-NCA with TMDA as an initiator. However, it is still quite probable that our samples so prepared were contaminated with molecules of the straight-rod type. Reduction of such contamination will be achieved by the choice of another solvent system for the polymerization.

Polarimetric methods are of little use for examining the molecular structures of PBG-C and PBG-D. We therefore employed the kinetic method for this purpose. At least from their kinetic behavior, it may be concluded that our PBG-C and PBG-D samples were block copolymers of the LD and LDL types, respectively. However, we have reason to believe that these samples, especially those of higher molecular weight, were also contaminated with homo-L and $D$ chains, though the extent could not be established.

Acknowledgement. We wish to thank Professor K. Kawahara, now at the School of Pharmacy of Nagasaki University, who helped us with the sedimentation experiments.

\section{REFERENCES}

1. A. Teramoto, T. Yamashita, and H. Fujita, J. Chem. Phys., 46, 1919 (1967).

2. R. D. Lundberg and P. Doty, J. Amer. Chem. Soc., 79, 3961 (1957).

3. W. B. Gratzer and P. Doty, ibid., 85, 1193 (1963).

4. W. E. Hanby, S. G. Waley, and J. Watson, J. Chem. Soc., 1950, 3009.

5. K. Nakagawa, K. Kawahara, A. Teramoto, H. Umaji, and H. Fujita, Rep. Progr. Polym. Phys. Japan, 11, 49 (1968).

6. E. R. Blout and R. H. Karlson, J. Amer. Chem. Soc., 78, 941 (1956).

7. M. Idelson and E. R. Blout, ibid., 79, 3948 (1957).

8. J. C. Mitchell, A. E. Woodward, and P. Doty, ibid., 79, 3955 (1957).

9. M. Szwarc, Advan. Polym. Sci., 4, 1 (1965).

10. Y. Imanishi, Kobunshi (High Polymers, Japan), 21, 32, 92 (1972).

11. A. Shoji and T. Kawai, Kobunshi Kagaku (Chem. High Polymers), 28, 805 (1971).

12. H. Fujita, A. Teramoto, K. Okita, T. Yamashita, and S. Ikeda, Biopolymers, 4, 769 (1966).

13. T. Norisuye, M. Matsuoka, A. Teramoto, and H. Fujita, Polymer J., 1, 691 (1970).

14. N. Nishioka, unpublished data.

15. D. G. H. Ballard and C. H. Bamford, Proc. Roy. Soc., A223, 495 (1954).

16. T. Matsumoto and N. Nishioka, unpublished data.

17. D. Poland and H. A. Scheraga, "Theory of Helix-coil Transition in Biopolymers," Academic Press, New York, N. Y., 1970.

18. M. Goodman, E.E. Schmitt, and D.A. Yphantis, J. Amer. Chem. Soc., 82, 3483 (1960); 84, 1288 (1962). 